\title{
UNIVERSITYOF BIRMINGHAM

\section{HLA DR2b-binding peptides from human endogenous retrovirus envelope, Epstein-Barr virus and brain proteins in the context of molecular mimicry in multiple sclerosis}

\author{
Ramasamy, Ranjan; Mohammed, Fiyaz; Meier, Ute-C
}

DOI:

10.1016/j.imlet.2019.10.017

License:

Creative Commons: Attribution-NonCommercial-NoDerivs (CC BY-NC-ND)

\section{Document Version}

Peer reviewed version

Citation for published version (Harvard):

Ramasamy, R, Mohammed, F \& Meier, U-C 2019, 'HLA DR2b-binding peptides from human endogenous retrovirus envelope, Epstein-Barr virus and brain proteins in the context of molecular mimicry in multiple sclerosis', Immunology Letters, vol. 217, pp. 15-24. https://doi.org/10.1016/j.imlet.2019.10.017

Link to publication on Research at Birmingham portal

\footnotetext{
General rights

Unless a licence is specified above, all rights (including copyright and moral rights) in this document are retained by the authors and/or the copyright holders. The express permission of the copyright holder must be obtained for any use of this material other than for purposes permitted by law.

- Users may freely distribute the URL that is used to identify this publication.

- Users may download and/or print one copy of the publication from the University of Birmingham research portal for the purpose of private study or non-commercial research.

- User may use extracts from the document in line with the concept of 'fair dealing' under the Copyright, Designs and Patents Act 1988 (?)

- Users may not further distribute the material nor use it for the purposes of commercial gain.

Where a licence is displayed above, please note the terms and conditions of the licence govern your use of this document.

When citing, please reference the published version.

Take down policy

While the University of Birmingham exercises care and attention in making items available there are rare occasions when an item has been uploaded in error or has been deemed to be commercially or otherwise sensitive.

If you believe that this is the case for this document, please contact UBIRA@lists.bham.ac.uk providing details and we will remove access to the work immediately and investigate.
} 
1 Title: HLA DR2b-binding peptides from human endogenous retrovirus envelope, Epstein-

2 Barr virus and brain proteins in the context of molecular mimicry in multiple sclerosis.

3 Authors: Ranjan Ramasamy ${ }^{1^{*}}$, Fiyaz Mohammed², Ute-C. Meier ${ }^{3}$

$4 \quad{ }^{1}$ ID-FISH Technology Inc., 556 Gibraltar Drive, Miltipas, CA 95035, United States of

5 America

$6 \quad{ }^{2}$ Cancer Immunology and Immunotherapy Centre, Institute of Immunology and

7 Immunotherapy, University of Birmingham, Edgbaston, Birmingham, B15 2TT, United

8 Kingdom

93 Department of Neuroscience and Trauma, Blizard Institute, 4 Newark St, Whitechapel, London E1 2AT, United Kingdom

* Corresponding author (RR)

Email: rir200911@yahoo.com

Running Title: Molecular mimicry in multiple sclerosis

Abbreviations: ABP - $\alpha, \beta$ Crystallin; $\beta S Y N$ - $\beta$ Synuclein; BLAST - Basic Local Alignment

Search Tool; CNS - Central Nervous System; EAE- Experimental Autoimmune

Encephalomyelitis; EBV - Epstein Barr Virus; EBNA1 - Epstein-Barr nuclear antigen 1; env

- envelope; HERV - Human Endogenous Retrovirus; IEDB - Immune Epitope Data Base;

MAG - Myelin-associated glycoprotein; MBP - Myelin Basic Protein; MOG - Myelin

Oligodendrocyte Glycoprotein; MS - Multiple Sclerosis; MSRV - Multiple Sclerosis

Associated Retrovirus; NCBI - National Center for Biotechnology Information; OSP _

Oligodendrocyte Specific Protein; PLP - Proteolipid Protein; SMM - Stabilised Matrix Method; SYN1 - Syncytin-1; SYN2 - Syncytin-2; TCR - T cell receptor. 


\section{Abstract}

Multiple sclerosis (MS) is a complex autoimmune disease in which T cells and antibodies damage the myelin sheath in the central nervous system. The aetiology of the disease is poorly understood. HLA Class II DR2b (DRB1*1501 $\beta$, DRA1*0101 $\alpha$ ) is the strongest genetic risk factor for MS. Genetic remnants of ancient retroviruses, termed human endogenous retroviruses (HERV) that have been incorporated into the human genome and Epstein-Barr virus (EBV) infection have also been associated with MS. In silico analyses of human endogenous retroviral envelope (HERV env) proteins and three myelin proteins (myelin basic protein, myelin oligodendrocyte glycoprotein and proteolipid protein) that are principal targets of the autoimmune response showed homologies between potential $T_{H}$ epitopes within pairs of viral and myelin peptides predicted to bind HLA DR2b. This led to the proposal that such molecular mimicry may potentially trigger MS. To further test this hypothesis, the HLA-DR2b binding characteristics of the three myelin proteins and HERV env peptides as well as in silico predicted peptides from other encephalitogenic brain proteins and EBV proteins were investigated. Peptides containing potential $T_{H}$ epitopes from the myelin oligodendrocyte glycoprotein and HERV env previously predicted to bind HLA DR2b as well as other pertinent potential HLA DR2b-restricted epitopes were shown to be able to do so in a cell-free binding assay. Molecular modelling of HLA-DR2b in complex with high affinity peptides derived from MOG and HERV env proteins highlighted that prominent surface exposed amino acids, which potentially interface with the T cell receptor, are conserved. A structurally similar pair of potential $T_{H}$ epitopes from the EBV protein EBNA1 and $\beta$ synuclein, a brain protein implicated in MS, were shown to be similarly capable of binding HLA DR2b molecules. Our findings justify future investigation of $T_{H}$ cell responses to the candidate peptides.

Key Words: autoimmunity; Epstein-Barr virus; HLA DR2b-peptide complex; human endogenous retroviruses; molecular mimicry; multiple sclerosis. 


\section{Introduction}

Multiple sclerosis (MS) is an inflammatory autoimmune disease of the central nervous system (CNS) that involves progressive damage to the myelin sheath and axons leading to neurodegeneration [1 - 3]. Studies on MS patients and experimental allergic (autoimmune) encephalomyelitis (EAE) in rodents have implicated several CNS proteins present in oligodendrocytes and myelin, including the myelin basic protein (MBP), myelin oligodendrocyte glycoprotein (MOG) and proteolipid protein (PLP), as targets of the autoimmune response in MS [1 - 3]. However, the aetiology of MS is not well understood. Cells of the innate immune system, $\mathrm{CD} 4^{+}$helper $\mathrm{T}$ cells, $\mathrm{CD} 8^{+}$cytotoxic $\mathrm{T}$ cells and autoantibodies are involved in the immunopathology of MS, while CD4 ${ }^{+}$helper 1 cells $\left(T_{H} 1\right)$ among all types of antigen-specific cells, are considered to play the critical role in initiating the autoimmune process [1 - 3]. Environmental factors, e.g. vitamin D deficiency [4], and infections especially with Epstein Barr virus (EBV) [1-2, 5-8], have been implicated in predisposition to MS. Genome-wide association studies demonstrate that the HLA Class II allele DRB1*1501 $\beta$ chain variant, which pairs with the relatively invariant DRA1*0101 $\alpha$ chain to form the HLA DR2b heterodimer in antigen-presenting cells (APCs), is the strongest genetic risk factor for MS [9]. The production of virions and expression of envelope protein (env) of a member of the genome-encoded human endogenous retrovirus W-family (HERVW), termed the MS- associated retrovirus or MSRV [10], has also been implicated in MS [11 - 15]. However, the molecular mechanisms linking $T_{H}$ cells to the genetic elements in the etiology of MS are not established. It has been recently hypothesized that epitopes in MSRV and other HERV family env proteins that cross-react with epitopes in myelin proteins, and presented by HLA DR2b on APCs to $T_{H} 1$ cells in an inflammatory milieu, provide the requisite link [16].

Sequence homologies have been demonstrated by BLAST analysis between MBP, MOG and PLP on one hand and MSRV env on the other $[16,17]$. In addition, structurally related sequences were found between the myelin proteins and syncytin-1 
(SYN1) [16], another HERV-W family-derived env protein that has evolved to perform an essential role in forming the syncytiotrophoblast of the placenta [18]. SYN1 is $87 \%$ identical in amino acid sequence to MSRV env [16] and also more distantly related to syncytin-2 (SYN2), another essential fusogenic placental protein derived from a different HERV family termed HERV-FRD [19]. SYN2 also possesses regions of amino acid sequence similarity with the three myelin proteins [16]. SYN1 has an additional fusogenic role in the development of myotubes from myoblasts [20] and possibly osteoclasts [21].

In silico analyses of myelin and HERV env sequences utilizing the Immune Epitope Data Base (IEDB) [22] to predict HLA DR2b-binding 15mer peptides showed homologies between potential nonamer $T_{H}$ epitopes within the 15mers from the HERV env proteins and all three myelin proteins that are predicted to bind to DR2b with high $\left(\mathrm{IC}_{50}<50 \mathrm{nM}\right)$ or intermediate affinity $\left(50 \mathrm{nM}<\mathrm{IC} \mathrm{C}_{50}<500 \mathrm{nM}\right)[16]$. Homologies between a potential nonamer epitope in MOG and those in MSRV env, SYN1 and SYN2 were particularly significant [16]. Interestingly, some predicted higher affinity DR2b-binding peptides lie within longer regions of sequence homology between myelin proteins and HERV env proteins whilst others do not [16]. Since SYN1 and SYN2 have evolved to perform essential physiological functions in humans, it is possible that $T_{H}$ cells that react with them may be deleted in the thymus and/or regulatory $T$ cells $\left(T_{\text {regs }}\right)$ that dampen an immune response are selected against them. This may not apply to MSRV env which is not expected to be normally expressed during development. However MSRV env is expressed within innate immune cells in an inflammatory situation e.g. during EBV infection [23] and is a potent stimulant of Toll-like receptor 4 present on macrophages and microglia, leading to impaired functional maturation of myelin-producing oligodendrocytes [24]. While existing data are consistent with an initiating role for molecular mimicry between the MSRV env and myelin proteins in MS, it is unclear whether this extends to the related SYN1 and SYN2 molecules. Once MS has been initiated in the proposed manner [16], further damage could 
arise from $T_{H}$ cells recognising other myelin epitopes presented by different HLA Class II molecules as a result of epitope spreading [16, 25].

This study experimentally investigated HLA DR2b binding of 15 mer peptides derived from MBP, MOG, PLP and HERV env proteins earlier identified in silico as potentially able to bind to HLA DR2b [16]. It also examined the binding to HLA DR2b of selected peptides from additional CNS proteins reported to be encephalitogenic [1] and corresponding structurally related peptides present in the three HERV env proteins as well as EBV proteins that have been described to elicit prominent human $\mathrm{CD}^{+}{ }^{+} \mathrm{T}$ cell responses [2]. The binding of selected peptides with high HLA DR2b binding affinity were additionally examined by molecular modelling of peptide-HLA DR2b complexes.

\section{Materials and Methods}

\subsection{Selection of CNS proteins for investigation}

The three myelin proteins previously used for in silico analysis of peptides capable of binding to HLA DR2b [16] and six other potentially encephalitogenic myelin and oligodendrocyte-associated CNS proteins $[1,26]$ selected for the present study are listed in Table 1.

\section{Table 1. CNS proteins selected for investigation}

\begin{tabular}{|l|l|l|}
\hline Protein & Abbreviation & NCBI sequence ID \\
\hline Myelin basic protein & MBP & P02686.3 \\
\hline Myelin oligodendrocyte glycoprotein & MOG & Q16653.2 \\
\hline Phospholipid protein & PLP & P60201.2 \\
\hline$\alpha, \beta$ Crystallin & ABP & ACP18852 \\
\hline Myelin-associated oligodendrocyte basic protein & MOPB & NP_001265251.1 \\
\hline Oligodendrocyte-specific protein & OSP & AAC25187 \\
\hline
\end{tabular}




\begin{tabular}{|l|l|l|}
\hline 2'3' Cyclic nucleotide 3' phosphodiesterase & CNPase & P09543 \\
\hline Myelin-associated glycoprotein & MAG & AAH53347.1 \\
\hline$\beta$-Synuclein & BSYN & Q16143 \\
\hline
\end{tabular}

\subsection{Selection of HERV and EBV proteins for investigation}

The three HERV env proteins used previously for predicting HLA DR2b-restricted peptides through the IEDB in silico procedure [16] and EBV proteins reported to elicit strong human $\mathrm{CD}^{+}{ }^{+} \mathrm{T}$ cell responses [2] were selected for the present study (Table 2).

Table 2. Virus-derived proteins selected for investigation

\begin{tabular}{|l|l|l|}
\hline Virus protein & Abbreviation & NCBI sequence ID \\
\hline HERV-W Syncytin-1 & SYN1 & Q9UQF0 \\
\hline HERV-FRD Syncytin-2 & SYN2 & NP_997465 \\
\hline $\begin{array}{l}\text { HERV-W Multiple sclerosis-associated retrovirus } \\
\text { envelope protein }\end{array}$ & MSRV env & AAK18189.1 \\
\hline Epstein-Barr nuclear antigen 1 & EBNA1 & YP_401677.1 \\
\hline Epstein-Barr nuclear antigen 2 & EBNA2 & ALV83014.1 \\
\hline Epstein-Barr nuclear antigen 3C & EBNA3C & CEQ33769.1 \\
\hline Epstein-Barr virus transactivator BZLF1 & BZLF1 & CAD53423 \\
\hline Epstein-Barr virus glycoprotein BZLF2 & BZLF2 & CEQ33770.1 \\
\hline Epstein-Barr virus envelope glycoprotein H & BXLF2 & ATE89094.1 \\
\hline
\end{tabular}

\subsection{Sequence homologies between CNS and virus-derived proteins}

The predicted additional CNS and virus-derived protein coding sequences obtained from the US National Center for Biotechnology Information (NCBI) data base were compared by pairwise Basic Local Alignment Search Tool (BLASTp) analysis online using default parameters (https://www.ncbi.nlm.nih.gov/blast), as previously described for MBP, 
MOG, PLP and the three HERV env proteins SYN1, SYN2 and MSRV env [16]. Additionally, every one of the selected CNS proteins shown in Table 1 were individually tested in BLASTp searches for homology against all non-redundant protein sequences of EBV (human herpes virus 4 strain B95-8) with NCBI taxonomy ID 10377.

\subsection{Prediction of peptides potentially binding to HLA DR2b molecules}

Prediction of potential peptides binding to HLA DR2b molecules was performed as previously described [13] using the IEDB analysis resource (www.iedb.org) [22, 27, 28]. The default peptide length of 15 amino acids was used in the analysis but the results also show the core nonamer peptides that are expected to bind to the HLA DR2b molecule and constitute the major portion of the T cell epitope [22, 27, 28]. The Stabilised Matrix Method (SMM) was used to rank the peptides according to their predicted binding affinities or $\mathrm{IC}_{50}$ which indicates the $\mathrm{nM}$ concentration of peptide expected to bind and achieve $50 \%$ saturation of the HLA DR2b molecules [22, 27, 28]. Structural similarities between core nonamer sequences in 15 mer peptides from different proteins that were predicted to bind $\mathrm{HLA}$ DR2b with high or intermediate affinity $\left(\mathrm{IC}_{50}<50 \mathrm{nM}\right)$ were determined manually.

\subsection{Determination of the binding affinity and stability of HLA DR2b-peptide complexes}

Peptides (15mers) were synthesized by FMOC solid-phase chemistry and quality checked with matrix assisted laser desorption ionization-time of flight mass spectrometry (MALDI-TOF MS) by Prolmmune (Oxford, UK). Binding characteristics of the peptides to HLA DR2b were determined by Prolmmune using the cell-free REVEAL® MHC class II binding assay [29]. The REVEAL $\AA$ assay measured the ability of a peptide to stabilize the MHC-peptide complex based on the detection of the native conformation of the MHC-peptide 
complex by a specific monoclonal antibody [29]. After an initial incubation with peptide for determining the proportion of $\mathrm{MHC}$ molecules binding the peptide (affinity), an additional measurement was taken after a further $24 \mathrm{~h}$ incubation at $37^{\circ} \mathrm{C}$ to determine the stability of binding (stability index). The stability index provides information on whether peptide can be presented long enough to serve as a T cell epitope. The affinity and stability index were measured as a percentage of the signal generated by the test peptide in comparison to a proprietary Prolmmune positive control peptide. A well characterised 15mer MBP peptide with the sequence ENPVVHFFKNIVTPR (hereafter referred to as MBP_3) that is presented by HLA DR2b and activates CD4+ $\mathrm{T}$ cells [30] was chosen as the internal comparative standard in the assays.

Details of the two sets of 40 peptides from CNS and viral proteins that were tested in the HLA DR2b binding assays are provided in Supplementary Table S1. The first set of 40 contained peptides derived from MBP, MOG and PLP and structurally similar peptides from SYN1 and MSRV env previously identified in silico as being potentially important for molecular mimicry by the IEDB algorithm [16]. Staggered arrays of 15 mers were used to identify the best binding peptide. The first set also contained a $15 \mathrm{mer}$ derived from EBV DNA polymerase shown to cross-react at the $\mathrm{CD}^{+} \mathrm{T}$ cell level with the control peptide MBP_3 on presentation by HLA DR2b [30]. A HLA DR2b-restricted MOG epitope shown previously to stimulate CD4 ${ }^{+}$T cells to produce IFNy [31] was also included in the first set of peptides. Other first set peptides were comprised of closely related signal sequence peptides of SYN1 and MSRV that encompassed structurally similar nonamers to those in internal peptides of MBP (including the control peptide MBP_3) and PLP [16], PLP peptides that contained the nonamer FFFLYGALL that were predicted to strongly bind DR2b [16], and four MSRV env peptides with the nonamer sequence TSVLVGPLV that exhibited weaker homology to MOG nonamer IVLPVLGPLV [16]. 
binding peptides identified from first set. They were independently synthesised and tested in REVEAL® binding assays. The second set additionally tested structurally similar pairs of HLA DR2b-binding peptides identified through IEDB in silico analysis in EBNA1 and HERV env proteins on one hand and different CNS proteins on the other. They included peptide pairs from EBNA1 and $\beta S Y N$, as well as EBNA1 and OSP, that had also been independently predicted to bind HLA DR2b using a different in silico algorithm [32]. The second set also included a different MBP peptide reported to be recognised by $T_{H}$ cells in the context of HLA DR2b [33].

\subsection{Modelling of 15mer peptides binding to HLA DR2b}

Molecular modelling of the HLA DR2b-peptide complexes were performed using the in silico docking program HADDOCK (high ambiguity driven protein-protein docking) [34]. Coordinates for the HLA DR2b complex were retrieved from the Protein Data Bank entry 1YMM [35]. Initial coordinates for the DR2b-restricted peptide moieties were extracted from the crystal structure of the T cell receptor(TCR)/HLA DR2b/MBP_3-peptide complex (entry 1YMM), and then used to build models of peptides with the molecular builder tool in COOT [36]. Each HLA DR2b-restricted peptide was subsequently subjected to a short regularisation protocol to ensure that the geometry of the peptide residues conformed to known bond lengths and angles.

The docking procedure was driven using only ambiguous intermolecular restraints, which were defined based on previously determined HLA DR2b-peptide complexes [35. 37, 38]. These structures revealed that the MBP_3 peptide is bound in the DR2b peptide-binding groove with peptide side chains P1, P4, P6 and P9 occupying pockets within the groove. Hence residues that line the P1, P4, P6 and P9 pockets of DR2b were selected as active residues (comprised of E11 $\alpha, F 24 \alpha, F 32 \alpha, W 43 \alpha, F 54 \alpha, N 62 \alpha, D 66 \alpha$, $R 76 \alpha, R 13 \beta, F 26 \beta, D 28 \beta, Q 70 \beta, A 71 \beta, Y 78 \beta, D 57 \beta$ and $W 61 \beta)$. For the peptide only the 
anchor residue side chains at P1, P4, P6 and P9 were defined as active residues. Passively involved residues were selected automatically. The 200 structures obtained after water refinement were analysed and ranked according to their HADDOCK score, a weighted sum of electrostatic, van der Waals, and restraint energy terms [34]. The lowest energy structure solutions were visualised and analysed using Pymol (The PyMOL Molecular Graphics System, Version 1.8 Schrödinger, LLC).

\section{Results}

\subsection{Sequence homologies between additional selected CNS and EBV or HERV env proteins}

Sequence homologies between the three HERV env proteins and the three myelin proteins MBP, MOG and PLP observed in BLASTp analysis have been previously described [16]. BLASTp analysis of the non-redundant protein sequences coded in whole EBV genome against each of the selected CNS proteins only revealed a weak homology between $\alpha, \beta$ crystallin (ABP) and a 53 residue segment of the EBV protein EBNA4 with an E value of 0.95 (Supplementary Table S2). Pairwise BLASTp analysis of each of the other selected CNS proteins against the three HERV env proteins demonstrated homologies with $E \leq 0.5$ only between the pairs ABP and SYN2, ABP and MSRV env, and myelin-associated glycoprotein (MAG) and MSRV env (Supplementary Table S3).

\subsection{Structurally related peptides in brain and EBV or HERV env proteins predicted to} bind to HLA DR2b molecules

IEDB analysis of 15 mer peptides containing structurally similar nonamers predicted to bind to HLA DR2b in MBP, MOG, PLP on one hand and the three HERV env proteins on the other, have been previously described [16]. Similar IEDB analysis performed 
on the determined structurally similar regions of ABP/SYN2, ABP/MSRV env and MAG/MSRV env (Supplementary Tables S2 \& S3) did not identify 15mer peptides of potentially high or intermediate affinity of binding to HLA DR2b that also contained structurally similar nonamer sequences in the three pairs of proteins (Supplementary Table S4 and reference 16).

Because of the homology observed between $\mathrm{ABP}$ and a 53 residue sequence of EBNA4 and perceived sequence similarities independently predicted between HLA DR2bbinding peptides of EBNA1 and several CNS proteins [32], HLA DR2b binding potential of 15mer peptides from EBNA1 and EBNA4 were also analysed by the IEDB procedure (Supplementary Table S5). These results when examined together with those in Supplementary Table S4 and data in reference 16 for HERV env, showed potential pairs of DR2b-binding peptides of high or intermediate affinity in EBNA1 and OSP, EBNA1 and BSYN, as well as OSP and MSRV env. These peptides whose sequences are given in Supplementary Table S6 were subsequently investigated in DR2b binding assays.

\subsection{Experimental binding to HLA DR2b of CNS and viral peptides predicted in silico to} bind HLA DR2b

The results of REVEAL binding assays on the selected peptides (Supplementary Table S6) showed that the pairs of peptides from MOG and the corresponding three HERV env proteins containing sequence-related nonamers previously predicted to engage HLA DR2b [16], and implicated in molecular mimicry, are able to bind HLA DR2b with comparable binding characteristics to the MBP_3 peptide.

The results also show that some OSP peptides with similar sequences to EBNA1 and MSRV env peptides and with predicted in silico intermediate binding affinity are able to bind well to HLA DR2b. However, the corresponding structurally related viral peptides did not reveal strong binding to HLA DR2b despite homology within the predicted nonamer 
sequences. For example, the OSP 15mer STTLRALAPRLMRRV which bound strongly had five identities in its predicted nonamer DR2b-binding sequence (LRALAPRLM) to the corresponding nonamer (LRALLARSH) in two 15mer EBNA1 peptides that however only showed weak binding to DR2b (Supplementary Table S6).

Peptides from the closely related signal sequences of SYN1 and MSRV that contained sequence-related nonamers to those in internal peptides of MBP (including the control peptide MBP_3) and PLP identified in the previous study [16] did not bind strongly to DR2b in the assays. Only one PLP peptide TASFFFLYGALLLAE that contained the nonamer sequence FFFLYGALL that was predicted to bind strongly to DR2b [16] was confirmed to bind strongly to DR2b. Four MSRV env peptides tested containing the nonamer sequence TSVLVGPLV with weaker homology to the MOG nonamer IVLPVLGPLV did not bind strongly to DR2b.

Peptides from EBV DNA polymerase and a different MOG region that had been shown to be presented on DR2b and stimulate $\mathrm{CD}^{+} \mathrm{T}$ cells $[30,31]$ revealed significant binding affinity to DR2b in the assay. A MBP peptide (GTLSKIFKLGGRDSR) containing a putative DR2b-resticted T cell epitope but with a weak predicted $\mathrm{IC}_{50}$ of $940 \mathrm{nM}$ based on IEDB analysis, only demonstrated marginal binding to DR2b.

An exact correlation between the in silico predicted affinity $\left(\mathrm{IC}_{50}\right)$ and the experimentally determined affinity by the REVEAL $\circledast$ binding assay for DR2b was not observed. For example, some PLP peptides with high predicted affinity $\left(\mathrm{IC}_{50}<1 \mathrm{nM}\right)$ showed poor experimental binding while four SYN2 peptides with predicted intermediate affinities $\left(\mathrm{IC}_{50}\right.$ of 130 to $\left.149 \mathrm{nM}\right)$ reveal experimental binding comparable to MBP_3 (Supplementary Table S6).

The details of binding assay results with staggered arrays of significant pairs of CNS and viral peptides are also shown graphically in Supplementary Table S6. Data on the 
best binding 15mer peptides with nonamers relevant for molecular mimicry grouped together and compared with the binding of the control MBP peptide are listed in Table 3.

Table 3. Binding characteristics and Haddock scores of the best pairs of DR2bbinding 15mer peptides containing sequence-related nonamers relevant to molecular mimicry

\begin{tabular}{|c|c|c|c|c|c|}
\hline $\begin{array}{c}\text { Homology } \\
\text { Group }\end{array}$ & Peptide & Peptide Sequence & $\begin{array}{c}\text { Relative } \\
\text { affinity }\end{array}$ & $\begin{array}{l}\text { Relative } \\
\text { stability }\end{array}$ & $\begin{array}{l}\text { HADDOCK } \\
\text { model score }\end{array}$ \\
\hline \multirow{4}{*}{$\begin{array}{l}\text { 1. MOG \& } \\
\text { HERV env }\end{array}$} & MOG_4 & ITLFVIVPVLGPLVA & 151 & 110 & $-123.7 \pm 2.5$ \\
\hline & MSRV env 5 & MPWTLPFLGPLAAII & 69 & 33 & $-158.1 \pm 2.1$ \\
\hline & SYN1 2 & MPWILPFLGPLAAII & 144 & 124 & $-152.5 \pm 4.4$ \\
\hline & SYN2 5 & KWFSWVLPLTGPLVS & 348 & 181 & $-137.8 \pm 5.3$ \\
\hline \multirow{3}{*}{$\begin{array}{l}\text { 2. } \beta S Y N \& \\
\text { EBNA1 }\end{array}$} & & & & & \\
\hline & $\beta$ synuclein & EKTKEGVLYVGSKTR & 91 & 95 & $-128.7 \pm 3.0$ \\
\hline & EBNA1_2 & VAGVFVYGGSKTSLY & 118 & 43 & $-131.5 \pm 5.1$ \\
\hline 3. Control & MBP 3 & ENPVVHFFKNIVTPR & 100 & 100 & $-163.7 \pm 1.6$ \\
\hline
\end{tabular}

295 Legend to Table 3. Results show the experimentally determined relative affinity and stability

296 of binding of peptides expressed as a percentage of that observed with the control MBP_3

297 peptide assigned values of 100 . The nonamers sequence predicted to bind in the peptidebinding groove in HLA DR2b are shown in bold letters and underlined. The docking scores

299 for the HADDOCK-derived lowest energy HLA DR2b-peptide complex models are shown.

\subsection{Molecular models of structurally similar peptides binding to HLA DR2b}

Previously identified HLA DR2b-restricted peptides of similar sequences

containing potential $T_{H}$ epitopes from MOG and HERV env were shown to bind HLA DR2b in the cell-free binding assay. We employed in silico molecular docking strategies to understand the molecular mechanisms governing binding of the structurally related pairs of peptides by HLA DR2b and their potential recognition by TCR. To evaluate the feasibility of 
ENPVVHFFKNIVTPR to HLA DR2b using HADDOCK and then compared with the available crystallographic structure (PDB entry $1 \mathrm{YMM}$ ) [35, 37, 38]. The HLA DR2b-MBP_3 complex model corresponding to the lowest intermolecular energy (with a HADDOCK score of -163.7) shows substantial similarity with the published structure in terms of epitope conformation and docking mode (Figure 1A). Superposition of the MBP_3 peptides derived from the published and model complex structures show that the main chain conformation is highly conserved (Figure 1A). In addition, similar to the published structure, the modelled MBP-3 peptide side chains at P1, P4, P6 and P9 serve as anchors slotting into the DR2b antigen binding cleft (Figure 1B\&C). Finally, in both the published and modelled complexes, the peptide was held in the DR2 antigen-binding cleft by a conserved network of hydrogen bonding and non-polar interactions (Figure 1B\&C). These observations justified the use of the HADDOCK docking approach to generate models of HLA DR2b bound to peptides that are relevant to MS.

To address the molecular mimicry hypothesis we generated models of HLA DR2b in complex with peptides of the highest affinity derived from MOG and the HERV env proteins MRSV env, SYN1 and SYN2 that are shown in Table 3. Superposition of the MOG, MSRV env, SYN1 and SYN2 peptides show that they all adopt a very similar back-bone conformation (Figure 2A). Similarly to the control MBP_3 peptide, the P1, P4, P6 and P9 peptide side chain positions serve as anchors inserting into the DR2b antigen binding cleft (Figure 2B-D). The HLA DR2b-peptide interactions were remarkably conserved between the different complexes including the control HLA DR2b-MBP_3 complex. In addition, positions P-1, P2, P5, and P8 are predicted to be surface exposed in the HADDOCK derived HLA DR2b-peptide complex models, and therefore potentially involved in binding to the TCR. The chemical characteristics of these prominent solvent exposed residues were either identical or structurally related in the relevant pairs of peptides. Taken together, these findings support the molecular mimicry hypothesis between MOG and HERV env proteins in triggering MS. 
To further test the molecular mimicry hypothesis, in silico predicted peptides from

other encephalitogenic brain proteins and EBV proteins were also investigated using modelling approaches. To address this HADDOCK derived models of HLA DR2b in complex with the $\beta S Y N$ and EBNA1 peptides shown in Table 3 were generated (Figure 3). These peptides adopted similar main chain conformations (Figure $3 \mathrm{~A}$ ) and mediated a conserved network of polar and non-polar interactions with side chains of DR2b (Figure 3 B\&C). As with comparisons between MOG and HERV env proteins, the most prominent surface exposed residues (at P-1, P2, P5, and P8) and hence potential TCR contacts were mainly conserved or semi-conservatively substituted between the $\beta S Y N$ and EBNA1 peptide pair. The nonanchoring residues were however different between the two sets of unrelated peptide pairs BSYN/EBNA1 and HERV env/MOG, and between each of these and MBP_3 (Table 3 and Figures 1-3).

The HADDOCK docking scores of the best binding 15mer peptides possessing the relevant structurally related nonamer pairs are listed in Table 3. The HADDOCK scores do not correlate with experimentally measured $\operatorname{REVEAL}{ }^{\circledR}$ binding affinities or stability indices for the peptides but the high negative values point towards energetically favourable binding to DR2b molecules.

\section{Discussion}

The molecular mimicry hypothesis proposed previously [16] attempted a unified explanation for the involvement of CD4+ $\mathrm{T}$ cells, HLA-DR2b, HERV env proteins and EBV infection in the origin of MS. It was supported by the in silico identification of structurally related pairs of 15 mer peptides predicted to bind DR2b in myelin-associated MBP, MOG and PLP proteins on one hand and HERV env proteins on the other. Homologies between predicted MOG and HERV env peptides were particularly prominent [16]. The present study extended the in silico predictions by examining the experimental binding of candidate $15 \mathrm{mer}$ 
peptides to DR2b as well as generating molecular models of such complexes. It also investigated the presence of potential DR2b-binding, structurally related, peptide pairs between HERV env and other CNS proteins as well as between CNS and EBV proteins.

More recent findings are pertinent to the original HERV-related molecular mimicry hypothesis. EBV, which primarily infects B cells, has been further implicated as a necessary but not sufficient cause of MS, partly because of its increased and dysregulated expression in peripheral blood and brain [39 -43]. In addition, antibody titres to EBNA1 have lately been confirmed to be higher in MS patients compared to controls [44]. EBNA1 has recently been reported to promote alternative splicing of cellular genes [45]. Since EBNA1 is widely expressed in EBV infected cells [46], it is intriguing to speculate that its splicing activity has a role in the trans splicing that has been postulated to produce functional MSRV env molecules [17]. This adds to the many different mechanisms proposed to explain why EBV infections are a predisposition for MS [1-2, 5-8].

HERVs and their putative role in autoimmunity have been lately reviewed [47-49] and cross-reactive B cell epitopes in MOG and HERV-W env have been documented [50]. The presence of antibodies to HERV-W env proteins have recently been reported to differentiate MS from related neurological diseases [51, 52].

Evidence that human GDP-L-fucose synthase peptides are recognised by CD4+ T cells in the context of HLA DRB3 ${ }^{*} 0202$ in MS patients, and that gut bacterial GDP-Lfucose synthase may be cross-reactive has led to a different proposal for molecular mimicry in MS [53]. RAS guanyl releasing protein 2 in peripheral memory B cells driving the proliferation of brain-infiltrating $C D 4^{+} T_{H} 1$ in a HLA DR2b-restricted manner that then recognise epitopes from the same protein expressed in brain cells has been proposed as another autoimmune mechanism explaining the association between MS and HLA DR2b [54]. 
myelinated axons in MS lesions, MSRV env induces inflammatory myelin and neuron damaging activity in vitro in microglia and that antibodies to MSRV can be neuroprotective in MS patients [12]. These observations are pertinent to further examining molecular mimicry between MSRV env and MOG.

The present study suggests that peptides containing nonamers with potential $\mathrm{T}$ cell epitopes in MSRV env, SYN1, SYN2 and MOG have the capacity to bind to HLA DR2b molecules with comparable affinities and similar binding topology to the well characterised MBP_3 peptide containing a T cell epitope. The molecular modelling suggests that potential surface exposed residues that contact TCR are relatively conserved between the MOG and HERV env peptides which is consistent with the proposed molecular mimicry hypothesis. The MOG peptides identified that possessed DR2b-binding nonamers that were related in sequence to those in the three HERV env proteins are located in the predicted $\mathrm{C}$ terminal transmembrane domain of MOG. The corresponding DR2b-binding nonamers of related amino acid sequence from MSRV env, SYN1 and SYN2 are also sited in predicted transmembrane domains. A longer peptide from the transmembrane region of MOG, that contained the MOG peptide identified in the present work, has independently been shown to stimulate $\mathrm{CD}^{+}{ }^{+} \mathrm{T}$ cells from MS patients to proliferate and secrete IFNy in a DRB-restricted manner [55]. It is possible that SYN1 and SYN2 may normally elicit tolerance as they may be recognised as self-proteins, while MSRV env may function as a foreign protein that can generate autoimmunity through molecular mimicry under certain circumstances as previously outlined [16]. Studies on CD4+ T cell response to the peptides identified in this study will help clarify the potential roles of MOG and the HERV env proteins in the immunopathogenesis of MS. It is relevant in this context that TCR recognition of MBP_3 bound to DR2b has been shown to involve skewed binding, not typical of TCR binding foreign peptide-Class II MHC complexes, which can result in potentially weaker interactions that may permit autoimmune T cells to escape deletion in the thymus [37]. 

epitopes between HERV env proteins and other encephalitogenic CNS proteins. This was also the case in our limited analysis of EBNA1 and EBNA4 against the selected CNS proteins except for a pair of sequence-related nonamers derived from $\beta S Y N$ and EBNA1 that showed binding affinity and stability comparable to MBP_3 in the REVEAL ${ }^{\circledR}$ assay. Modelling of the $\beta S Y N$ and EBNA1 peptides with HLA-DR2b revealed binding to the peptide binding cleft similar to MBP_3 and relative conservation of the surface exposed, potential TCR contact residues in the two peptides. This suggests the molecular mimicry is possible between $\beta S Y N$ and EBNA1. It is relevant in this context that $\beta S Y N$-reactive $T_{H}$ cells have recently been suggested to be responsible for autoimmune damage to CNS grey matter in the progressive stage of MS [26]. The possibility that EBNA1 generated, $\beta S Y N$-reactive $T_{H}$ cells induce additional autoimmune pathology, after the potential initiation of MS by molecular mimicry between MOG and HERV env proteins, therefore justifies investigation. Investigations on other pairs of potential HLA DR2b-binding peptides in EBNA1 and different CNS proteins predicted independently [32] may also be useful in this context as the present study was limited to CNS proteins with high encephalitogenic potential and restricted by the numbers of peptide pairs that could be studied in the HLA DR2b binding assay.

The HLA DR2a molecule is formed by pairing of the DRB5*0101 $\beta$ chain variant, whose gene is closely linked to the DRB1*1501 gene in many individuals, with the relatively non-polymorphic DRA $1^{*} 0101$ a chain. The previous in silico based predictions failed to identify strong DR2a binding pairs of potential sequence-related $T_{H}$ cell epitopes in HERV env and myelin proteins MBP, MOG and PLP [16]. However because of the close genetic linkage of the two $\beta$ chain loci, the investigation of potential DR2a binding structurally similar epitopes in the extended set of CNS proteins and EBV or HERV proteins is warranted because DR2a and DR2b molecules bind complementary sets of peptides through different binding motifs [56]. 
439

440

441

442

443

444

445

446

447

448

\section{Conclusions}

The results of the cell free HLA DR2b binding assays and molecular modelling show that sequence-related MOG and HERV env as well as $\beta S Y N$ and EBNA1 peptide pairs, with each set of pairs containing related potential $\mathrm{T}_{H}$ epitopes, are able to bind to HLA DR2b with similar affinity and conformation to a peptide MBP_3 containing an experimentally confirmed $\mathrm{T}_{\mathrm{H}}$ epitope. These findings support the previous in silico analysis-based prediction that pairs of sequence-related peptides in HERV env proteins and MOG are potential candidates for a molecular mimicry origin of MS. Kinetic studies of HLA DR2b binding with highly purified peptides and determination of the crystal structure of HLA DR2b-peptide complexes can provide more comprehensive binding information in the future. However, definitive support for molecular mimicry will require detailed studies on $C D 4{ }^{+} T_{H}$ cell responses to the candidate peptides characterised in this study. Such investigations may also contribute to the variety of immunomodulatory approaches presently being explored for treating MS [12, 24, $57-63]$.

\section{Conflict of interest statement}

The authors declare no conflict of interest.

\section{Acknowledgements}

This research was supported by the US National Multiple Sclerosis Society (PP1711-29350). FM is funded by the Wellcome Trust grant 099266/Z/12/Z. The authors are grateful to Professor Nick Willcox of the MRC Weatherall Institute of Molecular Medicine at the University of Oxford for his continuing encouragement.

\section{Author contributions}


RR and UM initiated the project, FM performed the modelling studies, and RR did the IEDB analysis, collation of data and drafting of the manuscript. All authors read and approved the final manuscript.

\section{References}

1. M. Sospedra, R. Martin. Immunology of multiple sclerosis. Annu. Rev. Immunol. 23 (2005) 683-747, doi: 10.1146/annurev.immunol.23.021704.115707.

2. G.S. Taylor, H.M. Long, J.M. Brooks, A.B. Rickinson, A.D. Hislop. The immunology of Epstein-Barr virus-induced disease. Annu. Rev. Immunol. 33 (2015) 787-821, doi: 10.1146/annurev-immunol-032414-112326.

3. C.A. Dendrou, L. Fugger, M.A. Friese. Immunopathology of multiple sclerosis. Nat. Rev. Immunol. 15 (2015) 545-558.

4. E. Kocovská, F. Gaughran, A. Krivoy, U-C Meier. Vitamin-D deficiency as a potential environmental risk factor in multiple sclerosis, schizophrenia, and autism. Front. Psychiatry 8 (2017) 47, doi: 10.3389/fpsyt.2017.00047.

5. M.F. Cusick, J.E. Libbey, R.S. Fujinami, Multiple sclerosis: autoimmunity and viruses, Curr. Opin. Rheumatol. 25 (2013) 496-501,

\section{http://dx.doi.org/10.1097/BOR.0b013e328362004d.}

6. J.S. Tzartos, G. Khan, A. Vossenkamper, M. Cruz-Sadaba, S. Lonardi, E. Sefia, et al., Association of innate immune activation with latent Epstein-Barr virus in active MS lesions. Neurology 78 (2012) 15-23, doi: 10.1212/WNL.0b013e31823ed057.

7. S. Sisay, L. Lopez-Lozano, M. Mickunas, A. Quiroga-Fernández, J. Palace, G. Warnes, et al., Untreated relapsing remitting multiple sclerosis patients show antibody production against latent Epstein Barr Virus (EBV) antigens mainly in the periphery and innate immune IL-8 responses preferentially in the CNS. J. Neuroimmunol. 306 (2017) 40-45, doi: 10.1016/j.jneuroim.2017.02.017.

8. S.V. Ramagopalan, R. Dobson, U.C. Meier, G. Giovannoni. Multiple sclerosis: risk 
factors, prodromes, and potential causal pathways. Lancet Neurol. 9 (2010) 727-739. doi: 10.1016/S1474-4422(10)70094-6.

9. International Multiple Sclerosis Genetics Consortium, Wellcome Trust Case Control Consortium, S. Sawcer, G. Hellenthal, M. Pirinen, C.C. Spencer, et al., Genetic risk and a primary role for cell-mediated immune mechanisms in multiple sclerosis, Nature 476 (2012) 214-219, http://dx.doi.org/10.1038/nature10251.

10. H. Perron, B. Lalande, B. Gratacap, A. Laurent, O. Genoulaz, C. Geny, et al. Isolation of retrovirus from patients with multiple sclerosis. Lancet 337 (1991) 862-863.

11. T. Christensen. Human endogenous retroviruses in the aetiology of MS. Acta. Neurol. Scand. 136 Suppl. 201 (2017) 18-21.

12. D. Kremer, J. Gruchot, V. Weyers, L. Oldemeier, P. Göttle, L. Healy, et al. pHERV-W envelope protein fuels microglial cell dependent damage of myelinated axons in multiple sclerosis. Proc. Natl. Acad. Sci. USA 116 (2019) 15216-15225. doi/10.1073/pnas.1901283116.

13. K.K. Nissen, M.J. Laska, B. Hansen, T. Terkelsen, P. Villesen, S. Bahrami, et al. Endogenous retroviruses and multiple sclerosis-new pieces to the puzzle. BMC Neurol. 13 (111) (2013), http://dx.doi.org/10.1186/1471-2377-13-111.

14. A. Dolei, E. Uleri, G. Ibba, M. Caocci, C. Piu, C. Serra. The aliens inside human DNA: HERV-W/MSRV/syncytin-1 endogenous retroviruses and neurodegeneration. J. Infect. Dev. Ctries. 9 (2015) 577-587, doi: 10.3855/jidc.6916.

15. E. Morandi, R.E. Tarlinton, B. Gran, Multiple sclerosis between genetics and infections: human endogenous retroviruses in monocytes and macrophages. Front. Immunol. 6 (647) (2015), http://dx.doi.org/10.3389/fimmu.2015.00647.

16. R. Ramasamy, B. Joseph, T. Whittall. Potential molecular mimicry between the human endogenous retrovirus $\mathrm{W}$ family envelope proteins and myelin proteins in multiple sclerosis. Immunol. Lett. 183 (2017) 79-85. dx.doi.org/10.1016/j.imlet.2017.02.003.

17. G.S. do Olival, T.S. Faria, L.H. Nali, A.C. de Oliveira, J. Casseb, J.E. Vidal, et al. 
Genomic analysis of ERVWE2 locus in patients with multiple sclerosis: absence of genetic association but potential role of human endogenous retrovirus type $\mathrm{W}$ elements in molecular mimicry with myelin antigen. Front. Microbiol. 4 (2013)172, doi: 10.3389/fmicb.2013.00172.

18. S. Mi, X. Lee, X. Li, G.M. Veldman, H. Finnerty, L. Racie, Syncytin-1 is a captive retroviral envelope protein involved in human placental morphogenesis. Nature 403 (2000) 785-789.

19. A.G. Lokossou, C. Toudic, B. Barbeau. Implication of human endogenous retrovirus envelope proteins in placental functions. Viruses 6 (2014) 4609-4627, http://dx.doi.org/10.3390/v6114609.

20. B. Bjerregard, I. Ziomkiewicz, A. Schulz, L.I. Larsson. Syncytin-1 in differentiating human myoblasts: relationship to caveolin-3 and myogenin. Cell Tissue Res. 357 (2014) 355-362, doi: 10.1007/s00441-014-1930-9

21. K. Søe, T.L. Andersen, A.S. Hobolt-Pedersen, B. Bjerregaard, L.I. Larsson, J.M. Delaissé. Involvement of human endogenous retroviral syncytin-1 in human osteoclast fusion. Bone 48 (2011) 837-846.

22. Y. Kim, J. Ponomarenko, Z. Zhu, D. Tamang, P. Wang, J. Greenbaum, et al. Immune epitope database analysis resource. Nucleic Acids Res. 40 (2012) W525-W530, doi: 10.1093/nar/gks438.

23. E. Morandi, R.E. Tarlinton, B. Gran. Multiple sclerosis between genetics and infections: human endogenous retroviruses in monocytes and macrophages, Front. Immunol. 6 (2015) 647, http://dx.doi.org/10.3389/fimmu.2015.00647.

24. A. Madeira, I. Burgelin, H. Perron, F. Curtin, A.B. Lang, R. Faucard. MSRV envelope protein is a potent, endogenous and pathogenic agonist of human toll-like receptor 4 : Relevance of GNbAC1 in multiple sclerosis treatment. J. Neuroimmunol. 291 (2016) 29-38, doi: 10.1016/j.jneuroim.2015.12.006

25. K. Raddassi, S.C. Kent, J. Yang, K. Bourcier, E.M. Bradshaw, V. Seyfert-Margolis, et al. Increased frequencies of myelin oligodendrocyte glycoprotein/MHC class II-binding 
CD4 cells in patients with multiple sclerosis, J. Immunol. 187 (2011) 1039-1046, http://dx.doi.org/10.4049/jimmunol.1001543.

26. D. Lodygin, M. Hermann, N. Schweingruber, C. Flügel-Koch, T. Watanabe, C. Schlosser C, et al. $\beta$-Synuclein-reactive T cells induce autoimmune CNS grey matter degeneration. Nature. 566 (2019) 503-508, doi: 10.1038/s41586-019-0964-2.

27. P. Wang, J. Sidney, C. Dow, B. Mothé, A. Sette, B. Peters. A systematic assessment of $\mathrm{MHC}$ class II peptide binding predictions and evaluation of a consensus approach. PLoS. Comput. Biol. 4 (2008) e1000048.

28. P. Wang, J. Sidney, Y. Kim, A. Sette, O. Lund, M. Nielsen, B. Peters. Peptide binding predictions for HLA DR, DP and DQ molecules. BMC. Bioinformatics 11 (2010) 568.

29. https://www.proimmune.com/ecommerce/page.php?page=reveal class2. Accessed 24 May 2019.

30. K.W. Wucherpfennig, J.L. Strominger. Molecular mimicry in T cell-mediated autoimmunity: viral peptides activate human T cell clones specific for myelin basic protein. Cell 80 (1995) $695-705$.

31. R. Weissert, J. Kuhle, K.L. de Graaf, W. Wienhold, M.M. Herrmann, C. Müller, et al. High immunogenicity of intracellular myelin oligodendrocyte glycoprotein epitopes. J. Immunol. 169 (2002) 548-556.

32. M. Tschochner, S. Leary, D. Cooper, K. Strautins, A. Chopra, H. Clark, et al. Identifying patient-specific Epstein-Barr NuclearAntigen-1 genetic variation and potential autoreactive targets relevant to multiple sclerosis pathogenesis. PLoS. One 11 (2016) e0147567, doi:10.1371/journal.pone.0147567

33. M. Pette, K. Fujita, D. Wilkinson, D.M. Altmann, J. Trowsdale, G. Giegerich, et al. Myelin autoreactivity in multiple sclerosis: recognition of myelin basic protein in the context of HLA-DR2 products by T lymphocytes of multiple-sclerosis patients and healthy donors. Proc. Natl. Acad. Sci. USA. 87 (1990) 7968-7672.

34. G.C.P. van Zundert, J.P.G.L.M. Rodrigues, M. Trellet, C. Schmitz, P.L. Kastritis, E. Karaca, et al. The HADDOCK2.2 web server: user-friendly integrative modeling of 
biomolecular complexes. J. Mol. Biol. 428 (2016) 720-725, doi:

$$
\text { 10.1016/j.jmb.2015.09.014. }
$$

35. M. Hahn, M.J. Nicholson, J. Pyrdol, K.W. Wucherpfennig. Unconventional topology of self peptide-major histocompatibility complex binding by a human autoimmune $\mathrm{T}$ cell receptor. Nat. Immunol. 6 (2005) 490-496.

36. P. Emsley, K. Cowtan. Coot: model-building tools for molecular graphics. Acta. Crystallogr. D Biol. Crystallogr. 60 (2004) 2126-2132.

37. M.J. Nicholson, M. Hahn, K.W. Wucherpfennig. Unusual features of self-peptide/MHC binding by autoimmune T cell receptors. Immunity 23 (2005) 351-360, doi:10.1016/j.immuni.2005.09.009.

38. K.J. Smith, J. Pyrdol, L. Gauthier, D.C. Wiley, K.W. Wucherpfennig. Crystal structure of HLA-DR2 (DRA*0101, DRB1*1501) complexed with a peptide from human myelin basic protein. J. Exp. Med. 188 (1998) 1511-1520.

39. C. Veroni, B. Serafini, B. Rosicarelli, C. Fagnani, F. Aloisi. Transcriptional profile and Epstein-Barr virus infection status of laser-cut immune infiltrates from the brain of patients with progressive multiple sclerosis. J. Neuroinflammation. 15 (2018)18, doi: 10.1186/s12974-017-1049-5.

40. M.P. Pender, P.A. Csurhes, J.M. Burrows, S.R. Burrows. Defective T-cell control of Epstein-Barr virus infection in multiple sclerosis. Clin. Transl. Immunology. 6 (2017) e126, doi: 10.1038/cti.2016.87.

41. A. Afrasiabi, G.P. Parnell, N. Fewings, S.D. Schibeci, M.A. Basuki, R. Chandramohan, et al. Evidence from genome wide association studies implicates reduced control of Epstein-Barr virus infection in multiple sclerosis susceptibility. Genome. Med. 11 (2019) 26, doi: 10.1186/s13073-019-0640-z.

42. B. Nourbakhsh, A. Rutatangwa, M. Waltz, M. Rensel, M. Moodley, J. Graves, et al. Heterogeneity in association of remote herpesvirus infections and pediatric MS. Ann. Clin. Transl. Neurol. 5 (2018)1222-1228, doi: 10.1002/acn3.636. 
602

43. A. Hassani, J.R. Corboy, S. Al-Salam, G. Khan. Epstein-Barr virus is present in the brain of most cases of multiple sclerosis and may engage more than just $B$ cells. PLoS. One 13 (2018) e0192109, https://doi.org/10.1371/journal.pone.0192109

44. S. Agostini, R. Mancuso, F.R. Guerini, S. D'Alfonso, C. Agliardi, A. Hernis, et al. HLA alleles modulate EBV viral load in multiple sclerosis. J. Transl. Med. 16 (2018) 80, doi: 10.1186/s12967-018-1450-6.

45. S. Boudreault, V.E.S. Armero, M.S. Scott, J.P. Perreault, M. Bisaillon. The EpsteinBarr virus EBNA1 protein modulates the alternative splicing of cellular genes. Virol. J. 16 (2019) 29, doi: 10.1186/s12985-019-1137-5.

46. J. McKenzie, A.G. El-Guindy AG. Epstein-Barr virus lytic cycle reactivation. Curr. Top. Microbiol. Immunol. 391 (2015) 237-261, doi: 10.1007/978-3-319-22834-1_8.

47. N. Grandi, E. Tramontano. HERV envelope proteins: physiological role and pathogenic potential in cancer and autoimmunity. Front. Microbiol. 9 (2018) 462, doi: 10.3389/fmicb.2018.00462.

48. V. Gröger, H. Cynis. Human endogenous retroviruses and their putative role in the development of autoimmune disorders such as multiple sclerosis. Front. Microbiol. 9 (2018) 265, doi: 10.3389/fmicb.2018.00265.

49. G. Morris, M. Maes, M. Murdjeva, B.K. Puri. Do human endogenous retroviruses contribute to multiple sclerosis, and if so, how? Mol. Neurobiol. 56 (2019) 2590-2605, doi: 10.1007/s12035-018-1255-x.

50. V. de Luca, A. H. Martins, C. M. Romano, G.M. Pimenta, L.A. Peroni. Cross-reactivity between myelin oligodendrocyte glycoprotein and human endogenous retrovirus $\mathrm{W}$ protein: nanotechnological evidence for the potential trigger of multiple sclerosis. Micron 120 (2019) 66-73, doi: 10.1016/j.micron.2019.02.005.

51. G. Arru, G. Mameli, G.A. Deiana, A.L. Rassu, R. Piredda, E. Sechi, et al. Humoral immunity response to human endogenous retroviruses $\mathrm{K} / \mathrm{W}$ differentiates between amyotrophic lateral sclerosis and other neurological diseases. Eur. J. Neurol. 25 (2018)1076-e84, doi: 10.1111/ene.13648. 
52. G. Arru, E. Sechi, S. Mariotto, A. Farinazzo, C. Mancinelli, D. Alberti, et al. Antibody response against HERV-W env surface peptides differentiates multiple sclerosis and neuromyelitis optica spectrum disorder. Mult .Scler. J. Exp. Transl. Clin. 3 (2017) 2055217317742425 , doi: $10.1177 / 2055217317742425$.

53. R. Planas, R. Santos, P. Tomas-Ojer, C. Cruciani, A. Lutterotti, W. Faigle, et al. GDPL-fucose synthase is a CD4+ T cell-specific autoantigen in DRB3*02:02 patients with multiple sclerosis. Sci. Transl. Med. 10 (2018) 462 pii: eaat4301, doi: 10.1126/scitransImed.aat4301.

54. I. Jelcic, F. Al Nimer, J. Wang, V. Lentsch, R. Planas, I. Jelcic, et al. Memory B cells activate brain-homing, autoreactive CD4+ T cells in multiple sclerosis. Cell 175 (2018) 85-100 e23, doi: 10.1016/j.cell.2018.08.011.

55. A. Shetty, S.G. Gupta, M. Varrin-Doyer, M.S. Weber, T. Prod'homme, N. Molnarfi, et al. Immunodominant T-cell epitopes of MOG reside in its transmembrane and cytoplasmic domains in EAE. Neurol. Neuroimmunol. Neuroinflamm. 1 (2014) e22, doi: $10.1212 / N X I .0000000000000022$.

56. E.M. Scholz, M. Marcilla, X. Daura, D. Arribas-Layton, E.A. James, I. Alvarez. Human leukocyte antigen (HLA)-DRB1*15:01 and HLA-DRB5*01:01 present complementary peptide repertoires. Front. Immunol. 8 (2017) 984, doi: 10.3389/fimmu.2017.00984.

57. C.A. Dendrou, L. Fugger. Immunomodulation in multiple sclerosis: promises and pitfalls. Curr. Opin. Immunol. 49 (2017) 37-43.

58. J. Chataway, K. Martin, K. Barrell, B. Sharrack, P. Stolt, D.C. Wraith, et al. Effects of ATX-MS-1467 immunotherapy over 16 weeks in relapsing multiple sclerosis. Neurology 90 (2018) e955-e962, doi: 10.1212/WNL.0000000000005118.

59. N. Kaushansky, A. Kaminitz, H. Allouche-Arnon, A. Ben-Nun. Modulation of MS-like disease by a multi epitope protein is mediated by induction of $C D 11 c+C D 11 b+G r 1+$ myeloid-derived dendritic cells. J. Neuroimmunol. 333 (2019) 476953. doi: 10.1016/j.jneuroim.2019.04.013. 
60. S. Kasagi, D. Wang, P. Zhang, P. Zanvit, H. Chen, D. Zhang, et al. Combination of apoptotic T cell induction and self-peptide administration for therapy of experimental autoimmune encephalomyelitis. EBioMedicine. (2019) S2352-3964(19)30306-8, doi: 10.1016/j.ebiom.2019.05.005

61. C.J. Pickens, M.A. Christopher, M.A. Leon, M.M. Pressnall, S.N. Johnson, S. Thati, et al. Antigen-drug conjugates as a novel therapeutic class for the treatment of antigenspecific autoimmune disorders. Mol. Pharm. 16 (2019) 2452-2461, doi: 10.1021/acs.molpharmaceut.9b00063.

62. N. Ji, A. Somanaboeina, A. Dixit, K. Kawamura, N.J. Hayward, C. Self, et al. Small molecule inhibitor of antigen binding and presentation by HLA-DR2b as a therapeutic strategy for the treatment of multiple sclerosis, J. Immunol. 191 (2013) 5074-5084, http://dx.doi.org/10.4049/jimmunol.1300407.

63. I. Zubizarreta, G. Flórez-Grau, G. Vila, R. Cabezón, C. España, M. Andorra, et al. Immune tolerance in multiple sclerosis and neuromyelitis optica with peptide-loaded tolerogenic dendritic cells in a phase 1b trial. Proc. Natl. Acad. Sci. USA. 116 (2019) 8463-8470, doi: 10.1073/pnas.1820039116.

\section{Figure Legends}

Figure 1 Comparison of the HLA DR2b-MBP_3 complex generated by HADDOCK with the reference structure. (A) Superposition of MBP_3 peptides bound to HLA $\mathrm{DR} 2 \mathrm{~b}$ in the reference (cyan) and modelled structures (black). (B) Ribbon representation of the published crystal structure of HLA DR2b bound to MBP peptide (MBP_3; ENPVVHFFKNIVTPR) (PDB entry 1YMM). (C) Ribbon representation of the lowest energy HLA DRb-MBP_3 complex model structure generated by HADDOCK. The HLA DR2b alpha and beta chains are depicted as pink and blue, respectively. For clarity only the peptide binding groove is highlighted. The peptide side chains (ball and stick format) and positions (red) are shown. Peptide residues P1, P4, P6 and P9 serve as anchor residues which slot into the antigen binding groove, whereas side 
chains at P-1, P2, P5 and P8 are surface exposed. HLA-DR2b residues involved in stabilising peptide binding are also highlighted (ball and stick format). The black rectangle boxes correspond to the core 9-mer sequence for each peptide. Figure was generated with Pymol (The PyMOL Molecular Graphics System, Version 1.8 Schrödinger, LLC)

Figure 2 Comparison of HADDOCK generated models of HLA-DR2b in complex with peptides derived from myelin (MOG) and HERV W-family (MSRVenv, SYN1 and SYN2) associated proteins. (A) Superposition of MOG_4 (red), MSRVenv_5 (blue), SYN1_2 (yellow) and SYN2_5 (green) peptides bound to HLA DR2b. (B) Ribbon representation of the lowest energy HLA DR2b-MOG_4 complex model structure. (C) Ribbon representation of the lowest energy HLA DRb-MSRV_5 complex model structure. (D) Ribbon representation of the lowest energy HLA DR2b-SYN1_2 complex model structure. (E) Ribbon representation of the lowest energy HLA DRbSYN2_5 complex model structure. The HLA DR2b alpha and beta chains are depicted as pink and blue, respectively. For clarity only the peptide binding groove is highlighted. The peptide side chains (ball and stick format) and positions (red) are shown. Peptide residues P1, P4, P6 and P9 serve as anchor residues which insert into the antigen binding groove, whereas side chains at P-1, P2, P5 and P8 are surface exposed. HLA-DR2b residues that contribute to peptide interactions are also highlighted (ball and stick format). The black rectangle boxes correspond to the core 9-mer sequence for each peptide.

Figure 3 Comparison of HADDOCK generated models of HLA-DR2b in complex with peptides derived from a CNS ( $\beta$-SYN) and an EBV (EBNA1) protein. (A) Superposition of $\beta S Y N$ (grey) and EBNA1_2 (orange) peptides bound to HLA DR2b. (B) Ribbon representation of the lowest energy HLA DR2b- $\beta S Y N$ complex model structure. (C) Ribbon representation of the lowest energy HLA DRb-EBNA1_2 
complex model structure. The HLA DR2b alpha and beta chains are depicted as pink and blue, respectively. For clarity only the peptide binding groove is highlighted. The peptide side chains (ball and stick format) and positions (red) are shown. Peptide residues P1, P4, P6 and P9 serve as anchor residues which slot into the antigen binding groove, whereas side chains at P-1, P2, P5 and P8 are surface exposed. HLA-DR2b residues involved in peptide binding are also highlighted (ball and stick format). The black rectangle boxes correspond to the core 9-mer sequence for each peptide. 